\title{
Female elk contacts are neither frequency nor density dependent
}

\section{Authors: P. C. Cross, T. G. Creech, M. R. Ebinger, K. Manlove, K. Irvine, J. Henningsen, J. Rogerson, B. M. Scurlock, \& S. Creel}

Copyright by the Ecological Society of America "This is the final version of record of an article that originally appeared in Ecology in September 2013.

Cross, PC, Creech, TG, Ebinger MR, Manlove K, Irvine K, Henningsen J, Rogerson J, Scurlock BM, Creel S. 2013. Female elk contacts are neither frequency nor density dependent. Ecology 94:2076-2086.

http://dx.doi.org/10.1890/12-2086.1

Made available through Montana State University's $\underline{\text { ScholarWorks }}$ 


\title{
Female elk contacts are neither frequency nor density dependent
}

\author{
P. C. Cross, ${ }^{1,8}$ T. G. Creeche, ${ }^{2,3}$ M. R. Ebinger,${ }^{2,4}$ K. Manlove, ${ }^{5}$ K. Irvine, ${ }^{1}$ J. Henningsen,,${ }^{6}$ J. Rogerson,${ }^{7}$ \\ B. M. Scurlock, ${ }^{7}$ and S. Creel $^{2}$ \\ ${ }^{1}$ U.S. Geological Survey, Northern Rocky Mountain Science Center. 2327 University Way, Suite 2, Bozeman, Montana 59715 USA \\ ${ }^{2}$ Montana State University, Department of Ecology, 310 Lewis Hall, Bozeman, Montana 59717 USA \\ ${ }^{3}$ Oregon State University, Department of Fisheries and Wildlife, 140 Nash Hall, Corvallis, Oregon 97331 USA \\ ${ }^{4}$ Montana State University, Institute on Ecosystems, 106 AJM Johnson Hall, Montana State University, \\ Bozeman, Montana 59717 USA \\ ${ }^{5}$ Pennsylvania State University, Department of Biology, Huck Institutes of the Life Sciences, 201 Life Sciences Building, \\ University Park, Pennsylvania 16802 USA \\ ${ }^{6}$ University of Wyoming, Wyoming State Veterinary Laboratory, 1174 Snowy Range Road, Laramie, Wyoming 82070 USA \\ ${ }^{7}$ Wyoming Game and Fish Department, 432 E Mill Street, Pinedale, Wyoming 82941 USA
}

Abstract. Identifying drivers of contact rates among individuals is critical to understanding disease dynamics and implementing targeted control measures. We studied the interaction patterns of 149 female elk (Cervus canadensis) distributed across five different regions of western Wyoming over three years, defining a contact as an approach within one body length $(\sim 2 \mathrm{~m})$. Using hierarchical models that account for correlations within individuals, pairs, and groups, we found that pairwise contact rates within a group declined by a factor of three as group sizes increased 33-fold. Per capita contact rates, however, increased with group size according to a power function, such that female elk contact rates fell in between the predictions of density- or frequency-dependent disease models. We found similar patterns for the duration of contacts. Our results suggest that larger elk groups are likely to play a disproportionate role in the disease dynamics of directly transmitted infections in elk. Supplemental feeding of elk had a limited impact on pairwise interaction rates and durations, but per capita rates were more than two times higher on feeding grounds. Our statistical approach decomposes the variation in contact rate into individual, dyadic, and environmental effects, and provides insight into factors that may be targeted by disease control programs. In particular, female elk contact patterns were driven more by environmental factors such as group size than by either individual or dyad effects.

Key words: brucellosis; Cervus canadensis; contact rate; disease models; elk; Greater Yellowstone Ecosystem, Wyoming, USA; hierarchical models; proximity loggers; super-spreading events; supplemental feeding.

\section{INTRODUCTION}

The relationship between host density and parasite transmission is fundamental to understanding infectious disease dynamics and implementing effective control strategies (Anderson and May 1991, McCallum et al. 2001). Models predict that when transmission is correlated with host density, parasites will be unable to persist when the host density is reduced below some threshold (Kermack and McKendrick 1927, Getz and Pickering 1983). In addition, culling is only expected to reduce disease prevalence when transmission is density dependent, which has important management implications for several wildlife diseases (Schauber and Woolf 2003, Lloyd-Smith et al. 2005a, Conner et al. 2007, Cross et al. 2010b). Defining the relationship between disease transmission and host density has been ham-

Manuscript received 29 November 2012; revised 19 March 2013; accepted 1 April 2013. Corresponding Editor: C. C. Wilmers.

${ }^{8}$ E-mail: pcross@usgs.gov pered by a paucity of data on host interaction rates across a range of densities and spatial scales.

In disease models, density-dependent (DD) transmission can be expressed as $\beta \pi S N$, where $\beta$ is the transmission coefficient, $\pi$ is the disease prevalence, $S$ is the density of susceptible individuals, and $N$ is the total population density. On the other hand, frequencydependent (FD) transmission is modeled as $\beta \pi S$. There are many alternatives to these two formulations (McCallum et al. 2001), but these two create a useful context in which we can place our empirical results. More generally, one could model transmission as power function $\beta \pi S N^{\kappa}$, where the force of infection is $\beta \pi N^{\kappa}$ for a given susceptible individual and $\kappa$ can be used to transition between DD and FD models. De Jong et al. (1995) pointed out that the origin of the term "mass action" assumes that the units of $S$ and $I$ are densities rather than numbers, and they introduced the term pseudo-mass action to refer to $\beta S I$ where $S$ and $I$ are numbers of individuals. In this paper, we use group sizes and number of individuals rather than densities, and are somewhat cavalier in our semantics referring to density- 
dependent and density-independent contact rates rather than pseudo-mass action contact rates.

Interactions among individuals are necessarily pairwise in contrast to most dynamic disease models that are parameterized on a per capita basis (but see Keeling 1999, Lloyd-Smith et al. 2004). In addition, nondirectional interaction data are more naturally analyzed on the basis of pairs rather than individuals because the same interaction would appear twice in an individuallevel data set. We can write the per capita encounter rate, irrespective of whether individuals are infectious (i.e., $\pi=1$ ), as $\varepsilon N^{\kappa}$, where $\varepsilon$ is a contact coefficient that does not account for the probability of infection, given contact. However, the per capita contact rate is not as easily observed and measured as the pairwise encounter rate, $\lambda$, which is $\varepsilon N^{\kappa}$ divided by the number of potential pairs an individual has, $N-1$, where $N$ is now the population or group size rather than density. Thus $\lambda$ is approximately $\varepsilon N^{\gamma}$, where $\gamma=\kappa-1$ for large $N$. When $\gamma$ equals zero or -1 , we recover the pairwise equivalents of the DD and FD transmission functions, respectively. In this study we illustrate how to directly estimate $\gamma$, and hence $\kappa$, from empirical interaction data while accounting for the repeated sampling of individuals and pairs over time and the correlation among pairs within a group.

There has been an extended debate about which disease transmission models are useful approximations of particular wildlife disease systems (McCallum et al. 2001, Begon et al. 2002, Schauber and Woolf 2003, Lloyd-Smith et al. 2005a). One challenge is that the adequacy of the model often depends upon spatial scale. Transmission may be density dependent at a local scale, but appear frequency dependent at a broad spatial scale (Turner et al. 2003, Cross et al. 2013). For socially aggregated species, this is likely to be true whenever disease transmission is closely related to local group size, but the frequency distribution of group size does not change with population size (Cross et al. 2009). Further, host density is a challenging variable to measure because it is often unclear what area should be in the denominator, and density measurements will therefore depend upon the spatial scale. For these reasons, we believe that models of interactions and transmission for socially aggregated species can be more easily connected to empirical data on group size, rather than densities. In this study, we measured how elk contact rates were affected by local group size and discuss our results in the broader context of density- and frequency-dependent transmission.

Few studies have directly estimated interaction rates across a range of host densities or population sizes, although several studies have related host density to some disease-related variable (for review see Ferrari et al. 2011) or have used indirect measures of contact from a coarse spatial or temporal scale, which may not correlate well with disease transmission (for a review see Cross et al. 2012). Ramsey et al. (2002) and Vander Wal et al. (2012) are two noteworthy exceptions. Ramsey et al. used radiotelemetry locations of brushtail possums (Trichosurus vulpecula) before and after density reductions to show that interaction rates were positively associated with host density, but that male-female interaction rates did not decrease in proportion to the decrease in density during the breeding season. Vander Wal et al. (2012) assessed elk interaction rates in enclosures of different sizes using proximity loggers, and found that male interaction rates increased with density, but interactions among females were unrelated to density. This experimental study controlled group size while modifying the area of the enclosure; however, it is not clear how to relate the results to a field setting where the area a group occupies is unconstrained and groups vary in size by more than an order of magnitude. In this study, we measured interaction rates at a fine spatial scale, $\sim 2 \mathrm{~m}$, in a field setting with 149 collared elk distributed across five different sites over three years with group sizes ranging from 10 to 336 (Fig. 1).

Elk in the Greater Yellowstone Ecosystem (GYE) are aggregated into groups from two to over 2000 individuals, which vary in size seasonally and spatially (Cross et al. 2010a). Within a season, factors that influence herd size include habitat type, habitat openness, and exposure to predation risk (Creel and Winnie 2005). In addition, many GYE elk are supplementally fed during winter at 22 feeding grounds in Wyoming, which affects aggregation patterns (Cross et al. 2007). In this region, elk are a reservoir host for brucellosis, a bacterial disease caused by $B$. abortus, which is a political and economic issue due to the potential transmission of brucellosis to cattle (Bienen and Tabor 2006). Our past work shows that recent increases in brucellosis seroprevalence among elk are correlated with increased elk density in many areas of the GYE (Cross et al. 2010a,b). However, the functional form of the relationship between seroprevalence and density was not well defined and the analyses were conducted at a relatively broad spatial scale.

Here we address one underlying mechanism that affects transmission and prevalence: elk interaction rates within groups. Disease ecologists often refer to contacts as interactions among individuals where pathogen transmission may occur even without physical contact. We refer to interactions and contacts interchangeably, but note that contacts do not necessarily imply physical touch. We use DD and FD models as two contrasting hypotheses about how pairwise and per capita interaction rates will correlate with group size. We also hypothesized that pairwise contact rates (interactions within $\sim 2 \mathrm{~m}$ ) may be higher during times when elk are supplementally fed, because artificial feeding on hay lines can cause elk to tightly aggregate in comparison to typical winter foraging behavior (Creech et al. 2012, Forristal et al. 2012). Finally, using a hierarchical approach, we illustrate how to partition the variation in the expected contact rates among individual, dyad, and environmental effects, which has important impli- 



FIG. 1. Map of the study area in northwestern Wyoming. Smaller dots (white and black) represent feeding grounds where elk (Cervus canadensis) are supplementally fed during winter months. Black dots are those areas where elk were collared with proximity loggers (SL, Soda Lake; FC, Fall Creek; MC, Muddy Creek; AL, Alpine). The large black circle (HA99) is the region without winter supplemental feeding.

cations for designing control efforts that attempt to target super-spreading events.

\section{Methods}

We conducted our study in the Wyoming and Wind River mountain ranges in the southern portion of the GYE (Fig. 1). In January and February of 2009, 2010, and 2011, we captured a total of 167 female elk ( $\geq 1.5 \mathrm{yr}$ old) at five sites and fitted them with Sirtrack proximity logger collars (Sirtrack, Hawkes Bay, new Zealand). Eighteen loggers were either not recovered or the data were corrupted, resulting in an average of 30 usable loggers at each site (Appendix A). Four sites had supplemental feeding during the winter (Soda Lake, Alpine, Muddy, and Fall Creek), and elk population sizes attending these feeding grounds ranged from 550 to 700 individuals. We captured elk at the site without supplemental feeding (elk hunt area 99) for two consecutive years (2010 and 2011; Fig. 1). Totals of
379 and 506 elk were counted during helicopter elk classifications conducted on winter ranges in hunt area $99\left(1782 \mathrm{~km}^{2}\right)$ during February in 2010 and 2011, respectively. We collared only adult female elk $(\leq 2 \mathrm{yr}$ old) because of our interest in brucellosis, which is thought to be primarily transmitted by abortion events from February to June (Cheville et al. 1998). We captured elk by chemical immobilization, helicopter net-gun, and corral traps. All captures were performed in accordance with approved Montana State University Animal Care and Use Protocol (no. 2010-2002).

It is difficult to interpret the mechanisms driving a lack of interaction among individuals using only the proximity logger data. In particular, one does not know whether noninteracting dyads from a given site and time period were separated by a vast distance and thus had no opportunity for contact, or if the dyad was in the same social group but remained outside of the distance required to $\log$ an interaction (Cross et al. 2012). Elk 


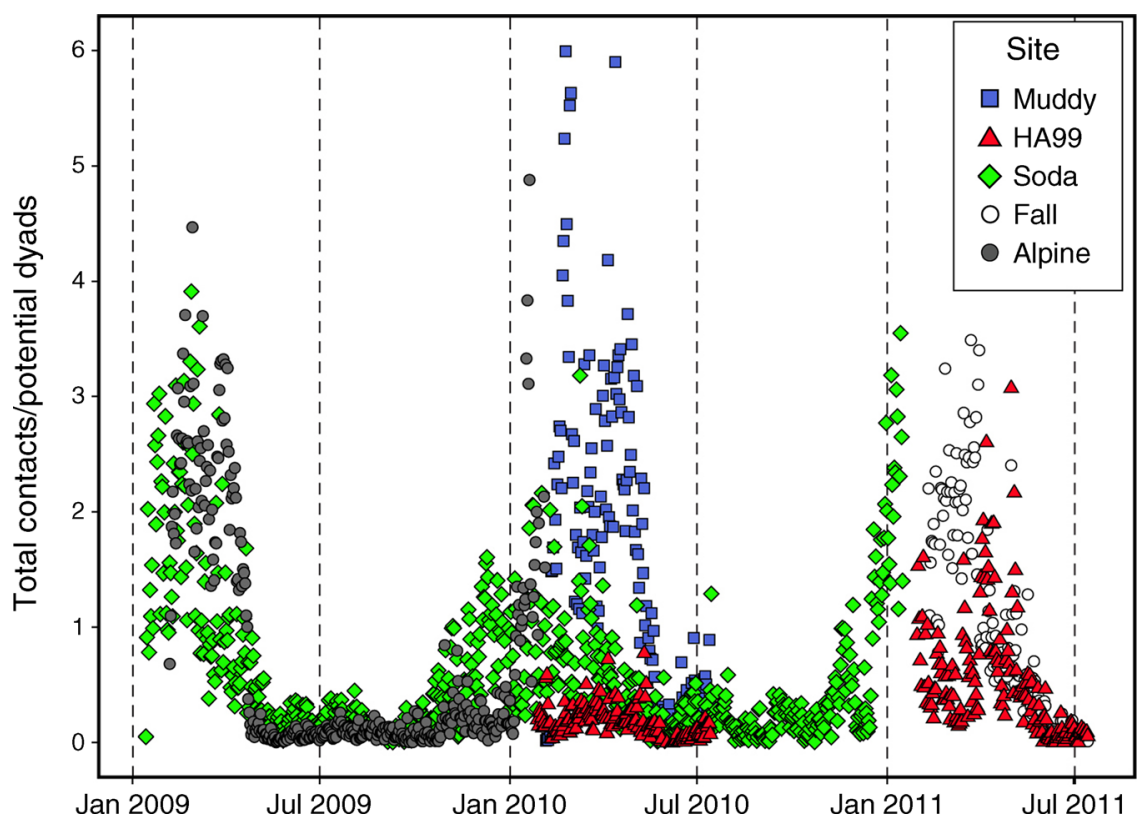

FIG. 2. Total number of contacts between female elk per day in a region divided by all potential dyads in that region. Potential dyads equal $n_{t}\left(n_{t}-1\right) / 2$ where $n_{t}$ is the number of individuals with a functional proximity logger in the region on day $t$.

tend to aggregate in winter and disaggregate into smaller groups in summer, so fewer recorded interactions during summer may be the result of having only a few proximity loggers in smaller groups rather than a change in the within-group behavior (Fig. 2). Therefore, we limited our analysis to days when group membership was known, which reduced the amount of data used in the analysis, but allowed us to interpret zero interactions as a failure to interact despite being in the same group. When we directly observed elk groups containing two or more proximity-collared individuals, we recorded the time, identity of collared individuals, and group size for each observation. At sites with supplemental feeding, we used contact data from January to March when all the elk with loggers were known to be present, based upon GPS collar data as well as visual inspection of pairwise contacts. This showed that all possible pairs of individuals were contacting one another almost every day during the feeding season (Appendix B; P. C. Cross and B. M. Scurlock, unpublished data).

We used a single count of the elk population at each feeding ground conducted in February, when the attendance at the feeding ground was highest. Daily counts of elk attending feeding grounds did not vary much over the time span we used for the analysis. While not attending feeding grounds, we delineated elk groups based upon relatively consistent internal spacing, whereby individuals were generally only a few body lengths away from one another, and individuals were moving in roughly the same direction (Winnie and Creel 2007). These groups were counted by direct observation using spotting scopes, which limited our analyses to open sagebrush habitats where the elk were most visible. We monitored elk from January to July each year, at which point the collars were programmed to drop off.

We calibrated proximity loggers to record interactions within $2 \mathrm{~m}$ in the field using a modified version of the laboratory calibration procedure described in Prange et al. (2006); the receiving range of each logger was tested using five other loggers to transmit signals, and we adjusted power settings on the receiving logger until its mean receiving range in the laboratory setting was as close to $3.5 \mathrm{~m}$ as possible. Our field tests, conducted with horses, revealed that a $3.5-\mathrm{m}$ laboratory receiving range was approximately equivalent to a $2-\mathrm{m}$ receiving range on the animal. Interactions were considered separate events if separated by $\geq 90$ seconds. We removed all contacts with a duration $\leq 1$ second as potentially spurious (Prange et al. 2006).

Analyzing association patterns is statistically complicated because interactions may be correlated within and among individuals, pairs, groups, and regions. We used Bayesian hierarchical generalized linear models to assess what factors are correlated with number of contacts while accounting for the repeated observations of some individuals, pairs, and multiple pairs observed within a group of a given size (Cross et al. 2012). Let $y_{l k}$ represent the number of contacts between dyad $l$ for observation period $k$, where $l$ is the unique dyad for individuals $i$ and $j$. We used a Poisson-Gamma mixture model formulation of the negative binomial model because the variance of the means of the posterior predictive distributions was roughly a quadratic function of the mean contact 
rate (Ver Hoef and Boveng 2007, Cross et al. 2012). Our initial model can be written as follows:

$$
\begin{aligned}
& y_{l k} \sim \operatorname{Poisson}\left(r_{l k} \lambda_{l k}\right) \\
& \lambda_{l k} \sim \exp \left(\alpha_{i}+\alpha_{j}+\delta_{l}+\rho_{k}\right) \\
& r_{l k} \sim \operatorname{Gamma}(\theta, \theta) \\
& \alpha_{i} \sim \operatorname{Normal}\left(0, \sigma_{\alpha}^{2}\right) \\
& \alpha_{j} \sim \operatorname{Normal}\left(0, \sigma_{\alpha}^{2}\right) \\
& \delta_{l} \sim \operatorname{Normal}\left(0, \sigma_{\delta}^{2}\right) \\
& \rho_{k} \sim \operatorname{Normal}\left(0, \sigma_{\rho}^{2}\right)
\end{aligned}
$$

where $\alpha_{i}$ and $\alpha_{j}$ are individual effects; $\delta_{l}$ are dyad effects (i.e., an interaction term, whereby some pairs may make more or less contacts than expected given their individual effects); and $\rho_{k}$ are effects associated with the observation period (e.g., group size), which we refer to as environmental effects. Each logger stores data on its own interaction history, so most contacts are recorded twice, once on each logger in the pair. We used data from the logger with the larger number of contacts recorded for the pair when loggers differed for a particular observation period. Each observation period $k$ represented a single observation of a group and contacts were summed for the $12 \mathrm{~h}$ before and after this observation. Our choice of a 24-h interval was motivated by the frequent switching of elk among groups; we did not want to assign individuals to the wrong group (Cross et al. 2012). Potential dyads that were never observed in the same group were excluded from the data analysis. Meanwhile, we added zeros to the data set when dyads were known to be in the same group from radiotelemetry or direct observations, but had no recorded interactions.

Proximity logger data include both the number of contacts and the duration of each contact. Total contact duration incorporates both of these, but it is complicated to statistically analyze because the distribution is likely to be bimodal, with a peak at zero (for those dyads that did not contact one another) and another peak at some average duration of contact. Thus, we conducted separate analyses of the number of contacts as well as the duration, $\tau_{l k}$, given that the pair made contact. We modeled contact duration as $\tau_{l k}=\exp \left(\alpha_{i}+\alpha_{j}+\delta_{l}+\rho_{k}\right)$ because the residuals (i.e., means of the posterior predictive distributions) were approximately normally distributed on a log scale. To calculate the expected per capita contact rate and duration, we multiplied the pairwise posterior means $\overline{\lambda_{\cdot k}}$ and $\overline{\tau_{\cdot k}}$ by $g_{k}-1$, where $g_{k}$ was the observed group size for observation period $k$. This accounts for the fact that not all individuals in a group are sampled. By formulating the statistical model on a pairwise basis and then translating those results to the per capita scale, we avoid the statistical issue on the per capita scale of having group size on both sides of the equation (e.g., $\lambda_{l k}\left[g_{k}-1\right]=\exp \left(\gamma \log \left[g_{k}\right]\right)$.

We were primarily interested in the effects of group size and supplemental feeding, and one potential hierarchical model is $\rho_{k} \sim \operatorname{Normal}\left(\phi_{\text {site }}+\phi_{\text {fed }}+\gamma \log \left[g_{k}\right]\right.$, $\sigma_{\rho}^{2}$ ), where $\phi_{\text {site }}$ and $\phi_{\text {fed }}$ are the main effects of site and feeding, and $\gamma$ is the effect of log group size. However, these predictors were correlated in our data set, such that during the feeding season, group sizes were larger and tended not to vary much over time. As a result, we could not assess the supplemental feeding effect independent of a group size effect. Thus we conducted one set of analyses using only observations collected after the feeding season when group sizes were known, and assumed that $\rho_{k} \sim \operatorname{Normal}\left(\phi_{\text {site }}+\gamma \log \left[g_{k}\right], \sigma_{\rho}^{2}\right)$. In a second analysis, we included data from during and after the supplemental feeding and used all observations where the group membership was known (by radiotelemetry) even when we did not have an estimate of the group size. For this data set, we estimated a parameter for each site both during and after the supplemental feeding season, $\rho_{k} \sim \operatorname{Normal}\left(\phi_{\text {site } \times \text { fed }}, \sigma_{\rho}^{2}\right)$, and then compared the sitelevel differences during and after feeding using posterior distributions of the linear contrasts.

Our statistical approach addresses several challenging problems that arise for valid inferences from dyadic data. First, each dyad involves two individuals, whose individual effect estimates may be assumed to derive from the same overall population (i.e., a single distribution). Second, the estimates of the variation associated with individuals, dyads, and environments are interesting in their own right; thus we would like to estimate the precision of those estimates. In particular, individual variation, $\sigma_{\alpha}^{2}$, relates to the $20 / 80$ rule of Woolhouse et al. (1997), who hypothesized that, in some cases, $20 \%$ of the individuals may be responsible for at least $80 \%$ of the infections. The estimate of $\sigma_{\alpha}^{2}$ is the predictable component of the variation among individuals; as $\sigma_{\alpha}^{2}$ increases, fewer individuals are involved in more of the interactions. Finally, because some of our individuals, dyads, or observation periods had only a few data points, the shrinkage toward the mean associated with our "random effects" produces better estimates with superior statistical properties than fixed effects (Efron and Morris 1977).

We used uninformative prior distributions on all parameters where possible. We assumed a diffuse normal prior distribution for site effects with a mean of 0 and a precision of $0.0001(\mathrm{SD}=100)$. We assigned the random effects $\alpha_{i}, \delta_{l}$, and $\rho_{k}$ normal prior distributions with a mean of 0 and a standard deviation with a hyperprior of Uniform $(0,10)$. The prior distribution for $\exp (\theta)$ was normal with a mean of 0 and a precision of 0.0001 . In previous analyses, we tested other forms of uninformative prior distributions, and our estimates were nearly identical (Cross et al. 2012). All models were run for 600000 iterations on three different 
Markov chains and the first half of each chain was discarded. We assessed convergence using the GelmanRubin-Brooks statistic, where $\hat{R}<1.1$ for all parameters indicated that relatively little variation was associated with a specific MCMC chain (Gelman and Hill 2007). All models were run using WinBUGS version 1.4.3 (Lunn et al. 2000) from $\mathrm{R}$ version 2.13.2 (R Development Core Team 2011). We were concerned that the sparse sampling of some pairs and groups may bias the group size parameter or the variance of the random effects. Therefore we repeated similar analyses using a Poisson linear mixed model using the lme4 package in $\mathrm{R}$ (Bates et al. 2011) and conducted simulations with known parameter values; we found no systematic bias for our sampling design (Appendix C).

After we recovered the proximity loggers from the field, we remeasured the distance at which they recorded contacts. Loggers, and pairs of loggers, differed from one another in the recording distance at the completion of the study, but this distance was not correlated with our estimated individual $\left(\alpha_{i}\right)$ or dyadic random effects $\left(\delta_{l}\right)$ in the contact analyses (data not shown). Further, our estimates of the differences among individuals, due to collar performance or other factors, were small; as a result, we ignored this complication. However, accounting for the performance of collars may be important to other studies interested in estimating the biological variation in the sociality of individuals.

\section{Results}

The full data set of contacts per dyad per day shows dramatic site and seasonal differences (Fig. 2). If we assume that all collared females within a site are available for contact, then average contact rates went as high as six times per pair per day during winter to less than 0.5 in the summer (Fig. 2). Data from Alpine and Soda Lake lasted longer because the collars did not fall off the animals as planned and individuals were recaptured in 2010 for Alpine and 2011 for Soda Lake. Supplemental feeding at Soda Lake occurred during the 2009 and 2011 winters, but not in 2010, primarily due to a lack of snow, which coincided with lower contact rates during the 2010 winter compared to 2009 and 2011. It is not clear from Fig. 2 if the seasonal variation in contact was a function of how the proximity loggers are distributed among groups or of behavioral changes within a group of a given size.

In our statistical analyses, we limited the data set to include only those dyads that were present within the same social group. Excluding data during the supplemental feeding season, the within-group pairwise contact rate declined with group size across all four sites for which we had group size observations $(\gamma=-0.38$; using lme4: $\gamma=-0.37, \mathrm{SE}=0.14)$. Pairwise contact rates declined by a factor of three, from about five to 1.4 contacts per day with a 33 -fold increase in group size (Fig. 3A). However, the corresponding per capita contact rates, $\overline{\lambda_{k}}\left(g_{k}-1\right)$, increased with group size from
45 contacts per individual per day in a group of 10 to over 400 contacts per individual per day in groups of 300 or more (Fig. 3B). Thus, as group size increased, the decrease in pairwise contact rates was more than offset by the increase in the number of possible dyads within the group. Pairwise duration together per day, given contact, $\tau_{l k}$, followed a similar pattern, declining from $\sim 7.4 \mathrm{~min}$ /day in the smallest groups to $1.2 \mathrm{~min} /$ day in the largest group, while the per capita contact duration increased from $1.2 \mathrm{~h} / \mathrm{d}$ to $6.7 \mathrm{~h} / \mathrm{d}$ (Fig. 3C, D). The 2.5th and 97.5th percentiles of the posterior distribution of $\gamma$ did not overlap either 0 or -1 for either the contact rate or contact duration analyses, suggesting that neither density-dependent (i.e., $\gamma=0$ ) nor density-independent (i.e., $\gamma=-1)$ models of per capita contact were supported (Table 1, Fig. 3).

We sampled at four sites (Alpine, Fall, Soda, and Muddy Creek) where elk were supplementally fed during winter, and we expected that the supplemental feeding would dramatically alter contact rates and duration of time spent together. This appears to be the case in Fig. 2, which implicitly assumes that all possible pairs at a given site could contact one another. On a pairwise basis, however, there was no clear pattern suggesting that supplemental feeding increases the per pair contact rate or duration (Fig. 4A, B). In particular, the unfed site (HA99), had contact rates and durations that were similar to those of feeding sites after the supplemental feeding had ended. Meanwhile, at Soda and Muddy Creek feeding grounds, the pairwise contact rates were slightly lower during the feeding season, while the opposite appears to be true for Fall Creek, although evidence for a significant statistical difference was not strong (Fig. 4). However, contacts and contact duration on the per capita scale were much higher during the supplemental feeding season (Fig. 4C, D). One might expect that more social individuals may be more likely to be exposed to infection. We found no relationship between brucellosis status and the estimate of that individuals' overall sociality, $\alpha_{i}$, for either contact rate or duration (data not shown).

The variation in observation periods, as measured by $\sigma_{\rho}^{2}$, was larger than either the individual $\sigma_{\alpha}^{2}$ or dyad $\sigma_{\delta}^{2}$ variation (Table 1). Our simulations of the statistical model suggest that these differences were not likely to be due to our observational sampling design (Appendix C). The variation among individuals, dyads, and observation periods was lower when we included data during the supplemental feeding season compared to after the feeding season (Table 1). As an example of how to interpret the estimated standard deviations in Table 1, consider just the estimates from the model of pairwise contact rate $\left(y_{l k}\right)$ using only the data after the supplemental feeding season. For this model, the average observation period effect $(\bar{\rho})$ was 0.92 , which we will use as the baseline. A dyad in which one elk had an individual effect $\left(\alpha_{i}\right)$ one standard deviation higher than average would be expected to interact 3.5 times per 
day $[\exp (0.92+0.33)=3.5]$ compared to an average of 2.5 times per day. Meanwhile, a pair of female elk with a dyad effect $\left(\delta_{l}\right)$ one standard deviation higher would interact 3.9 times per day. Finally, for an observation period that was one standard deviation higher than average, we would expect all pairs to interact, on average, 4.8 times per day, or almost twice as often as the baseline. Using data after the feeding season, the proportion of the variation in log contact duration explained by the random effects was relatively small $\left(\left[\sigma_{\alpha}^{2}\right.\right.$ $\left.+\sigma_{\delta}^{2}+\sigma_{\rho}^{2}\right] / \sigma^{2}=0.14 ;$ Table 1 )

\section{DisCuSSION}

Adult female elk interactions varied dramatically across sites, years, and seasons (Fig. 2). Each pair of individuals within a group was less likely to make contact in 24 hours, and the duration of contact decreased as group size increased (Fig. 3). These pairwise decreases were more than offset by the increasing number of pairs in larger groups, such that per capita contacts and durations increased with group size. Adult female elk interactions were intermediate to what might be expected for density- or frequencydependent disease models, where per capita contacts increase linearly with group size or are constant, respectively (Fig. 3). To our knowledge, this is the first study to directly estimate per capita and pairwise contact rates, of any species, across a wide range of group sizes.

Woolhouse et al. (1997) proposed a "20/80 rule" whereby $20 \%$ of individuals are responsible for $80 \%$ of disease transmission. Lloyd-Smith et al. (2005b) related this variation to super-spreading events and assessed how heterogeneity in pathogen transmission affects disease dynamics and the efficacy of control efforts. The variability in disease transmission is the product of variation in infectiousness, susceptibility, and contact rates among individuals and environments. However, only a portion of the variation in transmission and contacts is predictable, and in some cases it is only the predictable variation that can be targeted by control efforts (e.g., limiting large aggregations or targeting more sexually active individuals for a sexually transmitted infection). Here we illustrated an approach to assessing behavioral factors that are likely to create super-spreading events, using proximity loggers to measure interaction rates. Our statistical approach accounts for the dyadic nature of interactions and allows us to partition the predictable variation in contact rate into individual, dyad, and environmental effects. In our data set, environmental effects accounted for more variation in pairwise female contact rates than did either individual or dyad effects (Table 1). This suggests that identifying highly social individuals is less important than identifying the environmental conditions associated with high contact rates for disease control. By applying the approach we present here, in combination with quantitative measures of susceptibility and infec- tiousness, we can determine the drivers of superspreading events and develop targeted control measures even prior to a disease outbreak.

Proximity logger data are probably a useful surrogate for disease-relevant contacts for directly transmitted pathogens with limited survival in the environment. We focused our study on female elk over 1.5 years old, due to our interest in Brucella abortus, which is primarily transmitted by abortion events (Cheville et al. 1998). Placing a proximity logger under a fetus is a more direct measure in this host-parasite system (Creech et al. 2012). With the exception of the supplemental feeding grounds, however, it is difficult to place a fetus within free-ranging elk groups. We believe that elk-to-elk contact rates are a useful proxy for elk-fetus contacts within a group, particularly for retained placentas, which are periodically observed on the feeding grounds. Our focus on adult females, however, probably underestimates the total individual variation in contact rate for other diseases, such as tuberculosis, where males may play an important role and interact differently (Vander Wal et al. 2012). The amount of transmission between social groups due to the survival of B. abortus in the environment is unknown and not addressed in this study.

Contacts are, by definition, pairwise rather than individual events; however, disease models are typically formulated on a per capita basis. Translating between these two scales can result in counterintuitive results. For example, in our early statistical models we assumed a linear, rather than log-linear, effect of group size on pairwise interactions. If pairwise contact rates are statistically modeled as $\exp (\phi+\gamma N)$, then the per capita contact rate is approximately $N \exp (\phi+\gamma N)$. If $\gamma$ is negative, then this function is nonlinear and unimodal, with a maximum at intermediate population sizes. It is unlikely that per capita contacts would decline, rather than saturate, in the largest groups, but this is an important consequence of how the pairwise contacts are modeled. On the other hand, a pairwise contact rate modeled as $\exp (\phi+\gamma \log [N])$ becomes approximately $\exp (\phi) N^{\gamma+1}$ on the per capita scale. For our data set, linear or log-linear models of group size effects were not substantially different, but this may not be generally true.

Our results suggest that pairwise contact rates and durations were similar during and after the supplemental feeding season. On a per capita basis, however, contact rates were over two times greater during the feeding season. In this study we did not have data from unfed elk groups that were of equivalent size to the feeding ground populations, which creates potential confounding between group size and feeding effects. We addressed this by only estimating the group size effect using data after the supplemental feeding season had ended. The largest unfed groups had $\sim 300$ individuals, whereas the smallest feeding ground population was 420. The contact rate at that site (Muddy Creek) was roughly 

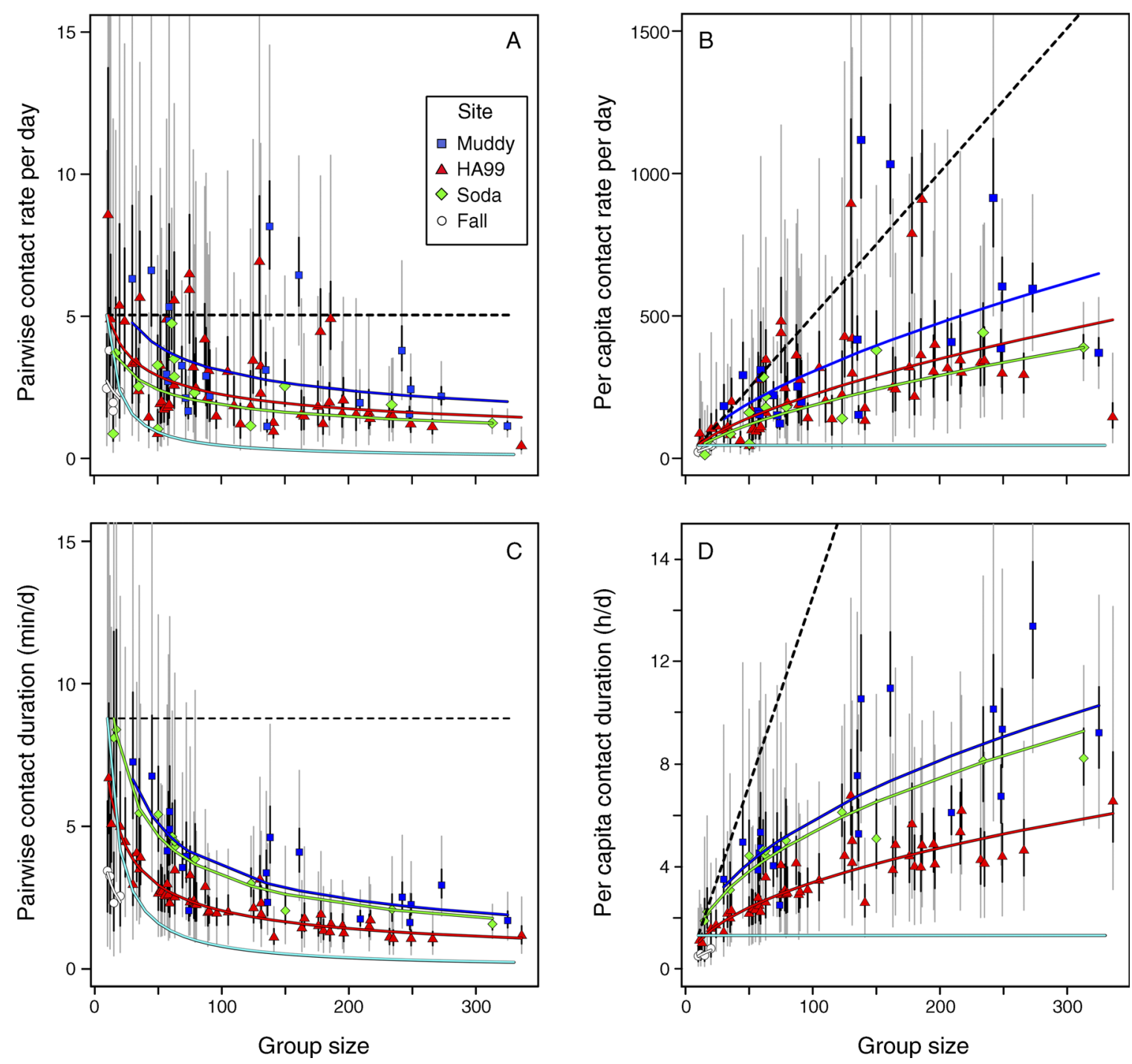

FIG. 3. (A, C) Expected adult female elk daily pairwise contact $(\mathrm{A})$ rates $\left(\overline{\lambda_{k}}\right)$ and $(\mathrm{C})$ durations, given contact $\left(\overline{\tau_{k}}\right)$ as a function of group size at four sites where groups were observed after the supplemental feeding season. (B, D) Per capita (B) contacts and (D) durations, given contact, are calculated as $\bar{\lambda}_{k}\left(g_{k}-1\right)$ and as $\bar{\tau}_{k}\left(g_{k}-1\right)$, respectively, where $g_{k}$ is the group size of group $k$. Points are the means of the posterior distributions, while the gray and black error bars are the $95 \%$ and $50 \%$ credible intervals. Curves represent the site-level model averages. The dashed black line represents a density-dependent model $(\gamma=0$, $\kappa=1)$, while the light blue line represents a density-independent model of per capita contact rate $(\gamma=-1, \kappa=0)$. The intercepts of the hypothetical models were not estimated from data but were set to 5 contacts/day and $7.4 \mathrm{~min} /$ day to facilitate comparison.

830 contacts per individual per day (Fig. 4), which is at least double the contact rate in the largest groups of unfed elk (Fig. 3), but only a $40 \%$ increase in group size. This suggests that supplemental feeding may increase per capita contact rates beyond what might be expected from the group size alone.

By limiting our analyses to just those days when group membership was known, we dramatically reduced the data available. In addition, our group size observations were limited to open habitats where groups could be counted. Pairing proximity loggers with global positioning systems (GPS) would allow future studies to use all of the available data and assess contact rates in areas, and at times, when individuals are not directly observable. The inclusion of more habitat-related variables may help to explain more of the variation in contacts, which in this study was relatively modest at $14 \%$ of the total variation in log contact duration (Table 1). We used 24 hours as the period of time to sum contacts and contact duration because elk switched group membership every few days. Longer time periods would have increased the chances of assigning individuals to the wrong group, resulting in lower contact rates. Longer time periods will be less problematic in systems where group membership is less dynamic. If the empirical data are going to be used in disease models, 
TABLE 1. Parameter estimates from statistical models of pairwise female elk (Cervus canadensis) contact rate and duration together per day.

\begin{tabular}{|c|c|c|c|c|c|c|}
\hline Dependent variable & Data set & Parameter & Mean & Median & $2.5 \%$ & $97.5 \%$ \\
\hline \multirow[t]{4}{*}{ Pairwise contacts per day $\left(y_{l k}\right)$} & all & $\sigma_{\alpha}$ & 0.28 & 0.28 & 0.24 & 0.33 \\
\hline & & $\sigma_{\delta}$ & 0.28 & 0.28 & 0.26 & 0.31 \\
\hline & & $\sigma_{\rho}$ & 0.55 & 0.55 & 0.47 & 0.58 \\
\hline & & $\theta$ & 0.97 & 0.97 & 0.95 & 0.99 \\
\hline \multirow{5}{*}{ Pairwise contacts per day $\left(y_{l k}\right)$} & after feeding & $\sigma_{\alpha}$ & 0.33 & 0.32 & 0.23 & 0.43 \\
\hline & & $\sigma_{\delta}$ & 0.45 & 0.45 & 0.33 & 0.57 \\
\hline & & $\sigma_{\rho}$ & 0.65 & 0.65 & 0.51 & 0.85 \\
\hline & & $\theta$ & 0.67 & 0.67 & 0.58 & 0.77 \\
\hline & & $\gamma$ & -0.38 & -0.37 & -0.64 & -0.09 \\
\hline \multirow[t]{4}{*}{ Duration per day $\left(\tau_{l k}\right)$} & all & $\sigma_{\alpha}$ & 0.24 & 0.24 & 0.20 & 0.28 \\
\hline & & $\sigma_{\delta}$ & 0.26 & 0.26 & 0.22 & 0.29 \\
\hline & & $\sigma_{\rho}$ & 0.26 & 0.26 & 0.22 & 0.31 \\
\hline & & $\sigma$ & 1.68 & 1.68 & 1.66 & 1.69 \\
\hline \multirow[t]{5}{*}{ Duration per day $\left(\tau_{l k}\right)$} & after feeding & $\sigma_{\alpha}$ & 0.33 & 0.33 & 0.21 & 0.38 \\
\hline & & $\sigma_{\delta}$ & 0.26 & 0.26 & 0.02 & 0.53 \\
\hline & & $\sigma_{\rho}$ & 0.40 & 0.39 & 0.21 & 0.60 \\
\hline & & $\sigma$ & 1.56 & 1.56 & 1.48 & 1.64 \\
\hline & & $\gamma$ & -0.52 & -0.53 & -0.78 & -0.26 \\
\hline
\end{tabular}

Notes: For the dependent variables, $l$ and $k$ are indices for the dyad and observation period, respectively. Parameters $\sigma_{\alpha}, \sigma_{\delta}$, and $\sigma_{\rho}$ are the standard deviations of the random effects of individuals, dyads, and observation periods, respectively; $\gamma$ is the effect of $\log$ (group size); and $\theta$ is the shape and scale of the Gamma distribution in the Poisson-Gamma mixture model.
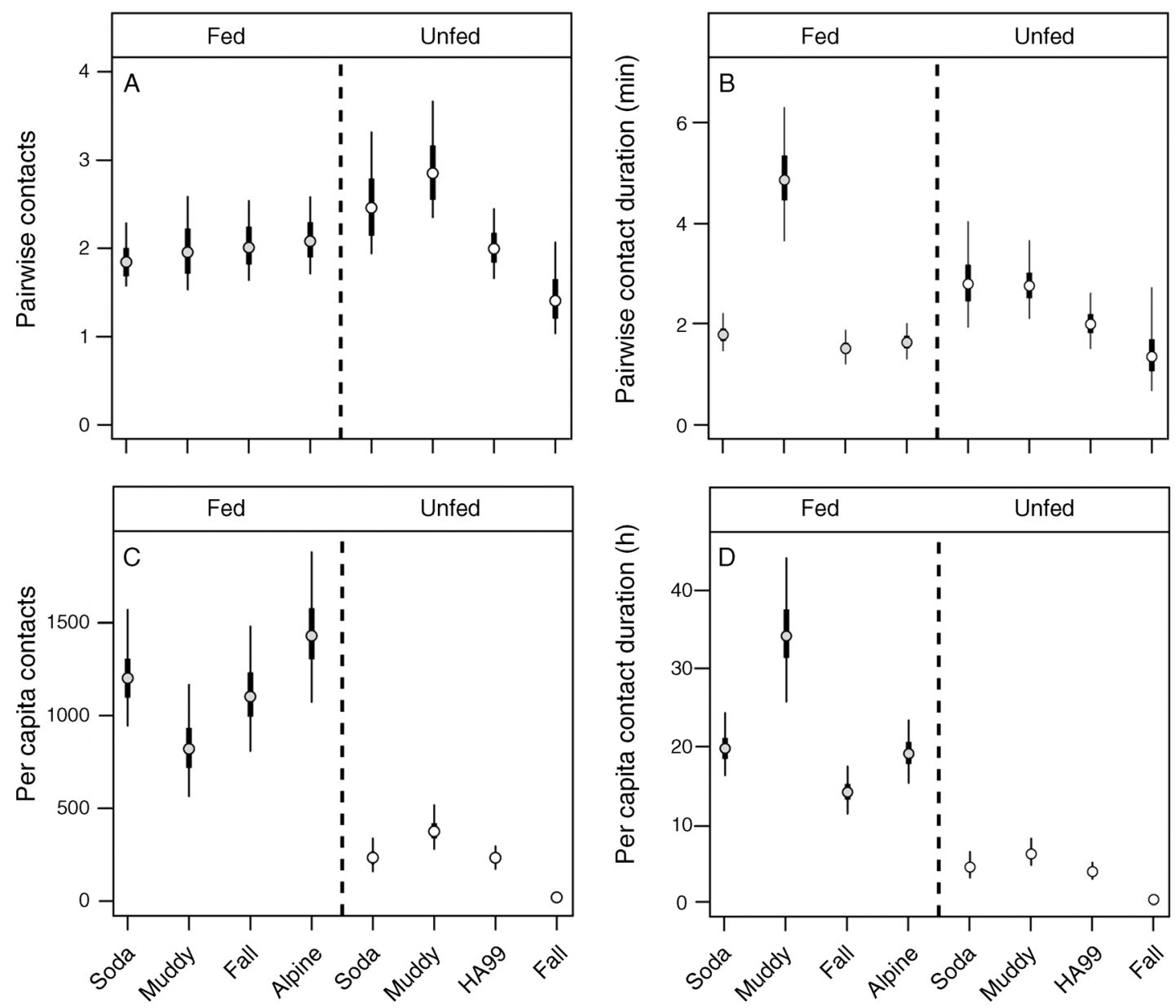

FIG. 4. (A, B) Comparisons of adult female elk pairwise (A) contact rates $(\bar{\lambda})$ and (B) durations $(\bar{\tau})$ at each site during and after the supplemental feeding season. (C, D) Per capita (C) contacts and (D) durations, given contact, were calculated as $\bar{\lambda}(\bar{g}-1)$ and as $\bar{\tau}(\bar{g}-1)$, respectively. Points represent means of the posterior predictive distributions. Thin and wide lines are the $95 \%$ and $50 \%$ credible intervals, respectively, some of which are hidden behind the points. 
the time period should be kept shorter than the infectious period.

Using time series data, Smith et al. (2009) found that cowpox prevalence in field voles (Microtus agrestis) was best fit by a function that was intermediate to density or frequency dependence. We expect this to be a general pattern for many host-pathogen systems because contacts, even nonsexual contacts, take time and time is limited (Antonovics et al. 1995). Our results suggest that, for a directly transmitted pathogen, we would expect the largest elk groups to play a disproportionate role in the disease dynamics for two reasons. First, by definition, more individuals will be in the largest groups. Secondly, on a per individual basis, contacts increase with group size, but at a decreasing rate. At broader spatial scales, brucellosis seroprevalence is potentially a nonlinear increasing function of elk density (Cross et al. $2010 a, b)$.

There does not appear to be much hierarchical structure among adult female elk within a group, nor would we expect many predictable super-spreaders in this system, based on their contact patterns. In our analyses, a cohesive group-within-group structure would be evidenced by a strong dyadic interaction effect, suggesting that some pairs contact often whereas others do not, even though they are all in the same group. If we had proximity loggers on mother-calf pairs, we probably would have observed such hierarchical structure. Instead it appears that adult female elk may be more random with their contacts, but that they interfere with one another as groups get larger, such that the number and duration of contacts with particular individuals declines with increasing group size. We hypothesize that this may be a general pattern for social ruminants, but not primates (Nunn and Altizer 2006) or elephants (Wittemyer et al. 2005), where hierarchies within groups are likely (Whitehead 2008).

\section{ACKNOWLEDGMENTS}

This work was supported by the Wyoming WildlifeLivestock Disease Partnership, National Science Foundation and National Institutes of Health Ecology of Infectious Disease Grant DEB-1 067 129, the Wyoming Game and Fish Department, and the U.S. Geological Survey. We thank E. Maichak, D. Damm, A. Williams, A. Barbknecht, and A. Roosa for their assistance in the field. Sky Aviation assisted with aerial flights. D. Heisey and two anonymous reviewers had many insightful suggestions on earlier drafts. Any use of trade, product, or firm names is for descriptive purposes only and does not imply endorsement by the U.S. Government.

\section{Literature Cited}

Anderson, R. M., and R. M. May. 1991. Infectious diseases of humans: dynamics and control. Oxford University Press, Oxford, UK.

Antonovics, J., Y. Iwasa, and M. P. Hassell. 1995. A generalized-model of parasitoid, venereal, and vector-based transmission processes. American Naturalist 145:661-675.

Bates, D., M. Maechler, and B. Bolker. 2011. lme4: Linear mixed-effects models using S4 classes. CRAN, R Project for Statistical Computing, Vienna, Austria. http://cran.r-project. org/web/packages/lme4/lme4.pdf
Begon, M., M. Bennett, R. G. Bowers, N. P. French, S. M. Hazel, and J. Turner. 2002. A clarification of transmission terms in host-microparasite models: numbers, densities and areas. Epidemiology and Infection 129:147-153.

Bienen, L., and G. Tabor. 2006. Applying an ecosystem approach to brucellosis control: can an old conflict between wildlife and agriculture be successfully managed? Frontiers in Ecology and the Environment 4:319-327.

Cheville, N. F., D. R. McCullough, and L. R. Paulson. 1998. Brucellosis in the Greater Yellowstone Area. National Academy Press, Washington, D.C., USA

Conner, M. M., M. W. Miller, M. R. Ebinger, and K. P. Burnham. 2007. A meta-BACI approach for evaluating management intervention on chronic wasting disease in mule deer. Ecological Applications 17:140-153.

Creech, T., P. C. Cross, B. M. Scurlock, E. J. Maichak, J. D. Rogerson, J. C. Henningsen, and S. Creel. 2012. Effects of low-density feeding on elk-fetus contact rates on Wyoming feedgrounds. Journal of Wildlife Management 76:877-886.

Creel, S., and J. A. Winnie. 2005. Responses of elk herd size to fine-scale spatial and temporal variation in the risk of predation by wolves. Animal Behaviour 69:1181-1189.

Cross, P. C., D. Caillaud, and D. M. Heisey. 2013. Underestimating the effects of spatial heterogeneity due to individual movement and spatial scale: infectious disease as an example. Landscape Ecology 28:247:257.

Cross, P. C., E. K. Cole, A. P. Dobson, W. H. Edwards, K. L. Hamlin, G. Luikart, A. D. Middleton, B. M. Scurlock, and P. J. White. 2010a. Probable causes of increasing elk brucellosis in the Greater Yellowstone Ecosystem. Ecological Applications 20:278-288.

Cross, P. C., T. G. Creech, M. R. Ebinger, D. M. Heisey, K. Irvine, and S. Creel. 2012. Wildlife contact analysis: emerging methods, questions, and challenges. Behavioral Ecology and Sociobiology 66:1437-1447.

Cross, P. C., J. Drewe, V. Patrek, G. Pearce, M. D. Samuel, and R. J. Delahay. 2009. Wildlife population structure and parasite transmission: Implications for disease management. Pages 9-30 in R. J. Delahay, G. C. Smith, and M. R. Hutchings, editors. Management of disease in wild mammals. Springer, Tokyo, Japan.

Cross, P. C., W. H. Edwards, B. M. Scurlock, E. J. Maichak, and J. D. Rogerson. 2007. Effects of management and climate on elk brucellosis in the Greater Yellowstone Ecosystem. Ecological Applications 17:957-964.

Cross, P. C., D. M. Heisey, B. M. Scurlock, W. H. Edwards, M. R. Ebinger, and A. Brennan. 2010b. Mapping brucellosis increases relative to elk density using hierarchical Bayesian models. PLoS One 5:e10322.

de Jong, M. C. M., O. Diekmann, and J. A. P. Heesterbeek. 1995. How does transmission of infection depend on population size? Pages 84-94 in D. Mollison, editor. Epidemic models: Their structure and relation to data. Cambridge University Press, Cambridge, UK.

Efron, B., and C. Morris. 1977. Stein's paradox in statistics. Scientific American 236:119-127.

Ferrari, M. J., S. E. Perkins, L. W. Pomeroy, and O. N. Bjørnstad. 2011. Pathogens, social networks, and the paradox of transmission scaling. Interdisciplinary perspectives on infectious diseases 2011:267049.

Forristal, V. E., S. Creel, M. L. Taper, B. M. Scurlock, and P. C. Cross. 2012. Effects of supplemental feeding and aggregation on fecal glucocorticoid metabolite concentrations in elk. Journal of Wildlife Management 76:694-702.

Gelman, A., and J. Hill. 2007. Data analysis using regression and multilevel/hierarchical models. Cambridge University Press, Cambridge, UK.

Getz, W. M., and J. Pickering. 1983. Epidemic models: thresholds and population regulation. American Naturalist 121:892-898. 
Keeling, M. J. 1999. The effects of local spatial structure on epidemiological invasions. Proceedings of the Royal Society of London Series B 266:859-867.

Kermack, W. O., and A. G. McKendrick. 1927. Contributions to the mathematical theory of epidemics. Proceedings of the Royal Society of Edinburgh 115:700-721.

Lloyd-Smith, J. O., P. C. Cross, C. J. Briggs, M. Daugherty, W. M. Getz, J. Latto, M. S. Sanchez, A. B. Smith, and A. Swei. 2005a. Should we expect population thresholds for wildlife disease? Trends in Ecology and Evolution 20:511519.

Lloyd-Smith, J. O., W. M. Getz, and H. V. Westerhoff. 2004. Frequency-dependent incidence in models of sexually transmitted diseases: portrayal of pair-based transmission and effects of illness on contact behaviour. Proceedings of the Royal Society of London B 271:625-634.

Lloyd-Smith, J. O., S. J. Schreiber, P. E. Kopp, and W. M. Getz. 2005b. Superspreading and the effect of individual variation on disease emergence. Nature 438:355-359.

Lunn, D. J., A. Thomas, N. Best, and D. J. Spiegelhalter. 2000. WinBUGS - a Bayesian modelling framework: concepts, structure, and extensibility. Statistics and Computing 10: 325-337.

McCallum, H., N. Barlow, and J. Hone. 2001. How should pathogen transmission be modelled? Trends in Ecology and Evolution 16:295-300.

Nunn, C. L., and S. Altizer. 2006. Infectious diseases in primates: behavior, ecology and evolution. Oxford University Press, Oxford, UK.

Prange, S., T. Jordan, C. Hunter, and S. D. Gehrt. 2006. New radiocollars for the detection of proximity among individuals. Wildlife Society Bulletin 34:1333-1344.

R Development Core Team. 2011. R: A language and environment for statistical computing. R Foundation for Statistical Computing, Vienna, Austria.

Ramsey, D., N. Spencer, P. Caley, M. Efford, K. Hansen, M. Lam, and D. Cooper. 2002. The effects of reducing population density on contact rates between brushtail possums: implications for transmission of bovine tuberculosis. Journal of Applied Ecology 39:806-818.

Schauber, E. M., and A. Woolf. 2003. Chronic wasting disease in deer and elk: a critique of current models and their application. Wildlife Society Bulletin 31:610-616.

Smith, M. J., S. Telfer, E. R. Kallio, S. Burthe, A. R. Cook, X. Lambin, and M. Begon. 2009. Host-pathogen time series data in wildlife support a transmission function between density and frequency dependence. Proceedings of the National Academy of Sciences USA 106:7905-7909.

Turner, J., M. Begon, and R. G. Bowers. 2003. Modelling pathogen transmission: the interrelationship between local and global approaches. Proceedings of the Royal Society of London B 270:105-112.

Vander Wal, E., H. Yip, and P. D. McLoughlin. 2012. Sexbased differences in density-dependent sociality: an experiment with a gregarious ungulate. Ecology 93:206-212.

Ver Hoef, J. M., and P. Boveng. 2007. Quasi-Poisson vs. negative binomial regression: How should we model overdispersed count data? Ecology 88:2766-2772.

Whitehead, H. 2008. Analyzing animal societies: quantitative methods for vertebrate social analysis. University of Chicago Press, Chicago, Illinois, USA.

Winnie, J., and S. Creel. 2007. Sex-specific behavioural responses of elk to spatial and temporal variation in the threat of wolf predation. Animal Behaviour 73:215-225.

Wittemyer, G., I. Douglas-Hamilton, and W. M. Getz. 2005. The socioecology of elephants: analysis of the processes creating multitiered social structures. Animal Behavior 69: $1357-1371$.

Woolhouse, M. E. J., et al. 1997. Heterogeneities in the transmission of infectious agents: Implications for the design of control programs. Proceedings of the National Academy of Sciences USA 94:338-342.

\section{Supplemental Material}

\section{Appendix A}

Table with covariate data on the female elk used in the analyses (Ecological Archives E094-189-A1).

\section{Appendix B}

Table with covariate data on the direct observations of elk groups used in the analyses (Ecological Archives E094-189-A2).

\section{Appendix C}

Details of a simulation study to assess the statistical properties of the hierarchical model with multiple random effects and sparse observational sampling (Ecological Archives E094-189-A3). 